\title{
Berezinskii-Kosterlitz-Thouless transition in two-dimensional dipolar stripes
}

\author{
Raúl Bombín ๑, ${ }^{*}$ Ferran Mazzanti, ${ }^{\dagger}$ and Jordi Boronat ${ }^{\ddagger}$ \\ Departament de Física, Universitat Politècnica de Catalunya, Campus Nord B4-B5, E-08034 Barcelona, Spain
}

(Received 24 July 2019; revised manuscript received 12 November 2019; published 5 December 2019)

\begin{abstract}
A two-dimensional quantum system of dipoles, with a polarization angle not perpendicular to the plane, shows a transition from a gas to a stripe phase. We have studied the thermal properties of these two phases using the path-integral Monte Carlo (PIMC) method. By simulating the thermal density matrix, PIMC provides exact results for magnitudes of interest such as the superfluid fraction and the one-body density matrix. As it is well known, in two dimensions the superfluid-to-normal phase transition follows the Berezinskii-Kosterlitz-Thouless (BKT) scenario. Our results show that both the anisotropic gas and the stripe phases follow the BKT scaling laws. At fixed density and increasing the tilting angle, the transition temperature decreases in going from the gas to the stripe phase. Superfluidity in the perpendicular direction to the stripes is rather small close to the critical temperature but it becomes larger at lower temperatures, mainly close to the transition to the gas. Our results are in qualitative agreement with the supersolidity observed recently in a quasi-one-dimensional array of dipolar droplets.
\end{abstract}

DOI: 10.1103/PhysRevA.100.063614

\section{INTRODUCTION}

The achievement of supersolidity as a new state of matter has been a longstanding topic since it was theoretically predicted in the 1960s of the past century [1]. A supersolid state is produced when two U(1) symmetries are simultaneously broken: the first one related to the presence of spatial long-range order and the second one to the emergence of a global phase giving rise to a superfluid state. The most natural candidate to be a supersolid is solid ${ }^{4} \mathrm{He}$, due to its extreme quantum character. However, and after a big excitement produced some years ago, the most accurate data available to date seem to exclude this possibility [2].

The difficulties in finding a stable condensed-matter supersolid state has moved its research to metastable systems which can exhibit the same properties. In recent years, the most fruitful tool to this end has been the versatile setup of ultracold quantum gases in the quantum degenerate regime. Although conventional dilute Bose Einstein condensate gases (BECs) do not break translational symmetry and thus are not good candidates for supersolid phases, some progress has been recently achieved by taking advantage of more exotic interactions. The first evidence of supersolidity came in 2017 almost simultaneously from two different experiments in reduced geometries. In the first one, a spin-orbit coupled system was shown to break translational symmetry in a twodimensional configuration [3] whereas, in the second one, this effect was achieved by coupling a Bose-Einstein condensate to the modes of two optical cavities [4]. Still in the context of ultracold gases, dipolar systems have been postulated as good

\footnotetext{
*raul.bombin@upc.edu

†ferran.mazzanti@upc.edu

¥jordi.boronat@upc.edu
}

candidates to the supersolid state. In fact, supersolid signatures have been observed by several groups [5-7], following previous theoretical work [8]. Recently, the gapless Goldstone excitation has also been measured for the same system [9-11].

In a previous work [12], the superfluid properties of the different phases of a dipolar system in two dimensions were studied at zero temperature. The stripe phase, that appears for certain densities and tilting angles, was shown to exhibit the characteristics which define a supersolid state. Recently, similar results have been reported for the equivalent system in the lattice [13]. At finite temperature, there is not condensate but quasicondensate reflected in an algebraic decay of the onebody density matrix. The transition between the superfluid (with quasi-off-diagonal long-range order) and normal phases follows the Berezinskii, Kosterlitz, and Thouless (BKT) theory $[14,15]$. This transition has been studied in many different systems such as helium films [16-18], Coulomb layers [19], and ultracold gases in pancake geometries [20-22]. It has also been shown that the BKT scenario stands even when disorder is introduced in the system [23,24].

In this paper, we study the superfluid-to-normal phase transition in a system of two-dimensional bosonic dipoles performing first principles path-integral Monte Carlo (PIMC) simulations. The particular case in which all the dipoles are polarized along the direction perpendicular to the plane, which constitutes the isotropic case, was already studied by Filinov et al. [25]. Here, we focus on the more general case in which dipoles are polarized in an arbitrary direction, within the stability limit, and show that the BKT scaling stands despite the anisotropy induced by the dipolar interaction. We determine the critical temperature $T_{\mathrm{BKT}}$ in both the gas and stripe phases. As schematically illustrated in Fig. 1, $T_{\mathrm{BKT}}$ for the stripe phase is smaller than $T_{\mathrm{BKT}}$ for the gas, at the same density. Increasing further the temperature, we observe that the normal stripes melt towards an anisotropic gas. 


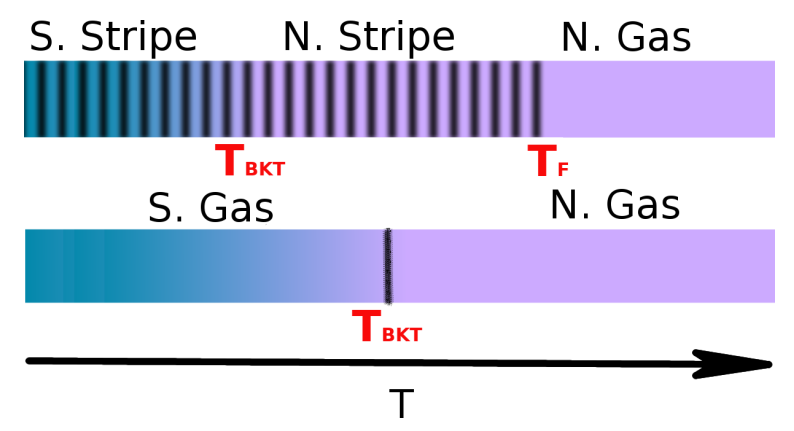

FIG. 1. Phase transitions in dipolar stripes compared with the dipolar gas. $\mathrm{N}$ and $\mathrm{S}$ labels stand for normal and superfluid phases, respectively.

\section{METHOD}

The system under study is composed of $N$ identical dipolar bosons of mass $m$ moving on the $X Y$ plane. An external field (electric or magnetic) in the $X Z$ plane polarizes all the dipoles along the same direction in space, forming an angle $\alpha$ with respect to the $Z$ axis. The model Hamiltonian describing the system reads

$$
H=-\frac{\hbar^{2}}{2 m} \sum_{j=1}^{N} \nabla_{j}^{2}+\frac{C_{d d}}{4 \pi} \sum_{i<j}^{N}\left[\frac{1-3 \lambda^{2} \cos ^{2} \theta_{i j}}{r_{i j}^{3}}\right],
$$

with $\lambda=\sin \alpha$ and $\left(r_{i j}, \theta_{i j}\right)$ the polar coordinates of $\mathbf{r}_{i j}$. The strength of the dipolar interaction is encoded in the constant $C_{d d}$ and is proportional to the square of the (electric or magnetic) dipole moment of each particle. Similar to previous works, we employ dipolar units [12,26], with the characteristic dipolar length $r_{0}=m C_{d d} /\left(4 \pi \hbar^{2}\right)$ and dipolar energy $\varepsilon_{0}=\hbar^{2} /\left(m r_{0}^{2}\right)$ that allows for writing the Hamiltonian in dimensionless units. In the following, temperatures will be expressed also in units of $\varepsilon_{0}$. The system is stable towards collapse as long as the tilting angle $\alpha$ is smaller than the critical value $\alpha_{c} \simeq 0.61$. Our simulations are carried out in a rectangular box, with periodic boundary conditions (PBC), to correctly commensurate the stripes [12], similar to what is made in the simulation of crystals.

For a given Hamiltonian, the PIMC method provides exact results (within some statistical noise) for the energy, structure, and superfluidity of a Bose fluid or solid. It has been widely used in the past to study the BKT transition, for instance, in two-dimensional liquid ${ }^{4} \mathrm{He}[17,27]$ and in dipoles with dipolar moments perpendicular to the plane [25]. Going down in temperature, and mainly close to the critical temperature, the PIMC simulation requires a good action to reduce the number of imaginary-time steps (beads) representing each atom (polymer) to a manageable level. To this end, we use the fourth-order Chin's action [28-31], that can be made to work effectively up to sixth order for the energy estimation by optimizing its control parameters [32]. Efficiency in the sampling of permutations is also fundamental to obtain accurate results for the one-body density matrix and superfluid densities. To get it right we use the worm algorithm, that has proven its accuracy in different systems [33].

At odds with what happens in three-dimensional systems, the superfluid fraction performs an abrupt universal jump
[34] at the critical temperature $T_{c}$. Near $T_{c}$, the BKT theory predicts that the correlation length has an essential singularity $\xi(T) \sim e^{a / t^{1 / 2}}$, with $t=\left(T / T_{c}-1\right)$ and $a$ being a nonuniversal parameter depending on density and on the microscopic properties of the particular system under study [35]. Due to the use of a finite number of particles $N$, within a finite-size box with PBC, we do not have direct access to the critical temperature in the thermodynamic limit $\left[T_{c}(\infty)\right]$ but rather to an estimation $T_{c}(L)$, with $L=\sqrt{N / n}$. As usual in finite-size scaling analysis of simulations close to the critical point, one identifies $T_{c}(L)$ with the temperature that makes $\xi\left(T_{c}(L)\right)=$ $L$. Therefore, the scaling law of the critical temperature with the size of the box can be written as [25]

$$
T_{c}(L)=T_{c}(\infty)+\frac{b}{\ln ^{2}(L \sqrt{n})},
$$

with $b$ a nonuniversal constant. On the other hand, the jump that the superfluid density performs at the critical temperature $T_{c}$ follows the universal relation [34]

$$
\frac{n_{s}\left(T_{c}, L\right)}{n}=\frac{2 m k_{B}}{\pi \hbar^{2}} \frac{T_{c}}{n}
$$

with $k_{B}$ the Boltzmann constant.

\section{RESULTS}

\section{A. Superfluid fraction}

In order to determine the critical temperature at which the superfluid-to-normal phase transition occurs, we need to evaluate the superfluid density. In the PIMC method, this is done through the well-known winding number estimator [36],

$$
\frac{n_{s}}{n}=\frac{m k_{B} T}{N \hbar^{2}}\left\langle\mathbf{W}^{2}\right\rangle
$$

where $\mathbf{W}$ is the winding number.

\section{BKT scaling of the gas phase}

Using the superfluid densities, calculated with the estimator (4) at different temperatures and system sizes, and taking advantage of the universal relations of Eqs. (2) and (3), one can obtain the superfluid-to-normal critical temperature. We start studying the transition in the gas phase at different densities and tilting angles. In Fig. 2, we show our PIMC results for the superfluid fraction $n_{s} / n$ at a density $n r_{0}^{2}=25$. In the left panel of this figure, we show our results for a tilting angle $\alpha=0.6$, close to the border of stability of the gas at zero temperature [37]. The critical temperature for a given system size $T_{c}(L)$ is determined as the crossing point between the universal BKT jump of Eq. (3) and the superfluid density for that system size. On the right panel of the same figure, we show how the scaling (2) is used to obtain the critical temperature in the thermodynamic limit. The analysis for different values of the tilting angle $\alpha=0,0.2,0.4$, and 0.6 reveals that the BKT scaling stands when anisotropy is present in the system. Our results for $\alpha=0$, corresponding to the isotropic gas, reproduce the PIMC estimations obtained by Filinov et al. [25]. In that work, a nonmonotonic behavior of the critical temperature as a function of the density [25] was found. The critical temperature, in units of density $T_{c} / n r_{0}^{2}$, increases at low densities and, above a characteristic value 

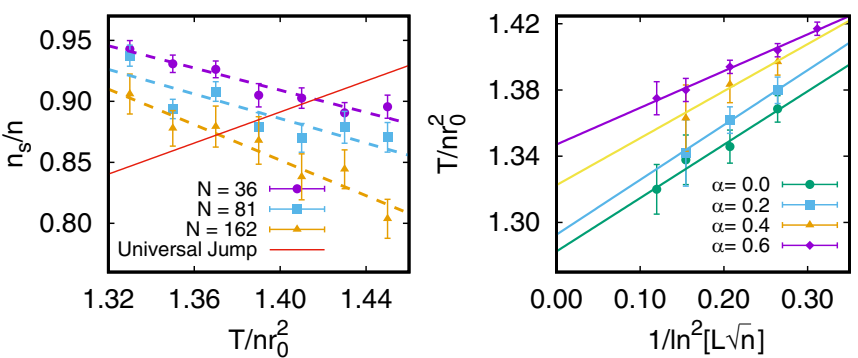

FIG. 2. Left panel: superfluid fraction as a function of temperature for different system sizes at density $n r_{0}^{2}=25$ and tilt angle $\alpha=0.6$. Points are MC results, dashed lines are linear fits to PIMC data, and the solid line is the universal jump of Eq. (3). The crossing points between the lines and the universal jump give the critical temperatures $T_{C}(L)$. Right panel: scaling of the critical temperature $T_{c}(L)$ with the system size, as given by Eq. (2), at the same density and for different polarization angles. Points are PIMC data and solid lines are linear fits.

$\left(1<n r_{0}^{2}<4\right)$, the behavior is the opposite. Filinov et al. [25] attribute this change to the appearance of the roton in the quasiparticle spectrum, which is observed to emerge around $n r_{0}^{2}=1[25,26,38]$. We have studied how the tilting angle $(\alpha>0)$ influences this behavior by calculating $T_{c}$ at low $\left(n r_{0}^{2}=0.01\right)$ and high $\left(n r_{0}^{2}=25\right)$ densities, as shown in Table I. The behavior of $T_{c} / n r_{0}^{2}$ with the tilting angle is the opposite for densities 0.01 and 25: increasing $\alpha$ reduces (increases) the critical temperature at low (high) density. In both cases, though, the growth of $\alpha$ translates into an effective reduction of the interaction strength since the $s$-wave scattering length for a given tilting angle is well approximated by [39]

$$
a_{s}(\lambda) \simeq e^{2 \gamma}\left(1-\frac{3 \lambda^{2}}{2}\right)
$$

with gamma the Euler's Gamma constant. According to Eq. (5), the scattering length for dipolar interaction decreases when $\alpha$ increases. In agreement with the isotropic case [25], the effective reduction of the interaction strength lowers $T_{c}$ at low densities, where the excitation spectrum is phononic, but increases it at high densities, when rotons dominate.

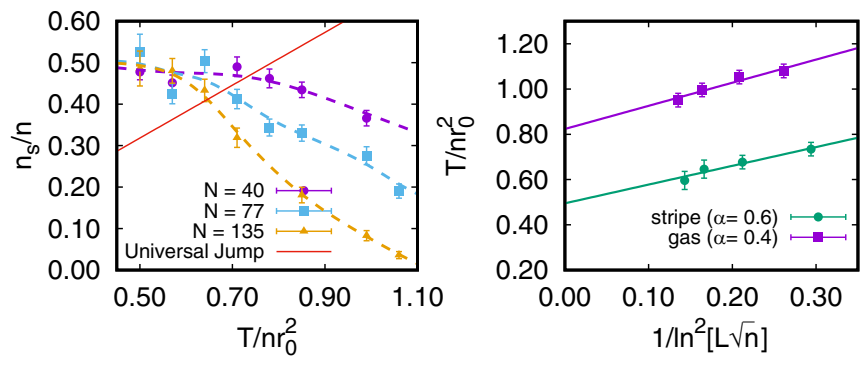

FIG. 3. Left panel: superfluid fraction as a function of the temperature for different system sizes, at density $n r_{0}^{2}=256$ and tilting angle $\alpha=0.6$, corresponding to the stripe phase. Points are PIMC results, dashed lines are guides to the eye, and the solid line is the universal jump (3). Right panel: scaling of the critical temperature $T_{c}(L)$ with the system size, as given by Eq. (2), at the same density and for two tilting angles: $\alpha=0.4$ (gas) and 0.6 (stripe). Points are PIMC data and solid lines are linear fits.

\section{BKT scaling of the stripe phase}

The stripe phase is of particular relevance in our study since it has been reported to be superfluid in the zerotemperature limit [12]. The simultaneous existence of spatial long-range order (in all but one direction of the space) and off-diagonal long-range order makes this phase close to the pursued supersolid state of matter. A relevant issue in this discussion is whether the BKT scaling, that we have shown to hold for the anisotropic gas, stands also for the stripe phase. In Fig. 3, we show PIMC results for the superfluid fraction at a density $n r_{0}^{2}=256$ and tilting angle $\alpha=0.6$, where the stripe phase is stable [12]. In the left panel, we show the behavior of the superfluid fraction as a function of temperature and for different number of particles in the simulation box. As in the gas phase, the crossing of this line with the universal jump law of Eq. (3) allows us to extract the critical temperature for a given system size $T_{c}(L)$. In the right panel, we compare the scaling of these critical temperatures for the stripe phase with the ones obtained for the same density but at a smaller tilting angle $\alpha=0.4$, where the gas phase is the stable one. As one can see, the BKT scaling holds in both cases, and thus one can apply it to estimate the critical temperature in the thermodynamic limit.

TABLE I. BKT critical temperatures (in dipolar units) for different values of the density $n r_{0}^{2}$ and tilting angle $\alpha$, in both the gas and stripe phases. The superfluid fraction at the critical temperature is evaluated through Eq. (3). Figures in parentheses are the estimated errors.

\begin{tabular}{|c|c|c|c|c|c|c|c|}
\hline \multicolumn{8}{|c|}{ Gas phase } \\
\hline$\overline{n r_{0}^{2}}$ & $\alpha$ & $T_{c} / n r_{0}^{2}$ (units of $\varepsilon_{0}$ ) & $n_{s} / n\left(T_{c}\right)$ & $n r_{0}^{2}$ & $\alpha$ & $T_{c} / n r_{0}^{2}$ (units of $\varepsilon_{0}$ ) & $n_{s} / n\left(T_{c}\right)$ \\
\hline 0.01 & 0.0 & $1.316(6)$ & $0.838(4)$ & 25 & 0.0 & $1.282(8)$ & $0.816(6)$ \\
\hline 0.01 & 0.2 & $1.317(3)$ & $0.838(6)$ & 25 & 0.2 & $1.292(5)$ & $0.823(4)$ \\
\hline 0.01 & 0.4 & $1.29(11)$ & $0.821(6)$ & 25 & 0.4 & $1.322(1)$ & $0.842(3)$ \\
\hline 0.01 & 0.6 & $1.263(13)$ & $0.804(8)$ & 25 & 0.6 & $1.347(3)$ & $0.858(2)$ \\
\hline 128 & 0.4 & $1.04(4)$ & $0.66(3)$ & 256 & 0.4 & $0.82(3)$ & $0.52(2)$ \\
\hline \multicolumn{8}{|c|}{ Stripe phase } \\
\hline 128 & 0.6 & $0.60(7)$ & $0.38(4)$ & 256 & 0.6 & $0.49(4)$ & $0.31(3)$ \\
\hline
\end{tabular}


One could think that the stripe phase is composed of quasione-dimensional channels, which dominate the superfluid signal, in such a way that the superfluidity in stripes follow the one-dimensional scaling law instead of the BKT one. In the next section we show that this is not the case, and thus only the BKT scenario is plausible with our results (see Sec. III A 3).

For temperatures lower than $T_{c}$, the superfluid fraction shows a plateau around a value which is in agreement with the zero-temperature result derived previously using the diffusion Monte Carlo method [12], $\left[\frac{n_{s}}{n}\right]_{\alpha=0.6}^{n r_{0}^{2}=256}=0.54(5)$.

In Table I, we report the results for the critical temperature and superfluid fraction at $T_{c}$ of the stripe phase with $\alpha=0.6$ and densities $n r_{0}^{2}=128$ and 256. By increasing the density, the critical temperature in the stripe phase decreases in a similar form to what has been previously obtained for the gas at high density. However, if the tilting angle increases, at fixed density, and crosses from the gas to the stripe phase both the superfluid fraction and the critical temperature decrease (see, for instance, data at $n r_{0}^{2}=128$ in Table I). In other words, superfluidity in stripes is thermally more fragile than in the gas phase. The winding number estimator for superfluidity (4) can be split into the $X$ and $Y$ directions corresponding to the stripe orientation and its perpendicular one, respectively. At $T_{c}$, the superfluid fraction in the $Y$ direction for a finite $N$ value is $<5 \%$ and decreases with $T$ faster than the one along the stripe direction. As it was observed previously [12], the superfluidity across the stripes depends strongly on the tilting angle, keeping the density fixed, reaching values $\sim 100 \%$ close to the gas-stripe phase transition line but decreasing fast when entering the deep stripe region.

\section{Non-Luttinger liquid behavior of the stripe phase}

One may wonder if the stripe phase at finite temperature might be considered as an ensemble of one-dimensional systems. If this were the case, our data should accommodate the predictions of the Luttinger liquid (LL) theory. Although one-dimensional systems do not show superfluidity in the thermodynamic limit, one can still see a nonzero superfluid fraction in a finite system of length $L$. For a one-dimensional liquid, described by Luttinger theory, the superfluid fraction for a Galilean invariant system is predicted to scale with the system size as [40]

$$
\frac{n_{s}}{n}=\frac{\gamma}{4} \frac{\left|\Theta_{3}^{\prime \prime}\left(0, e^{-\gamma / 2}\right)\right|}{\Theta_{3}\left(0, e^{-\gamma / 2}\right)}
$$

where $\Theta_{3}(z, q)$ is the Theta function, $\Theta_{3}^{\prime \prime}(z, q)=$ $d^{2} \Theta_{3}(z, q) / d z^{2}$, and $\gamma=\frac{m k_{B} T L}{\hbar^{2} n_{l}}$ with $n_{l}$ the linear density.

In Fig. 4 , we show that the data for the stripe phase $\left(n r_{0}^{2}=\right.$ 128 and $\alpha=0.6$ ) do not collapse to a single line when doing the scaling with $\gamma$, with a lineal density $n_{l}=14.6(3)$ obtained from $n_{l}=N /\left(L N_{s}\right)$ with $N_{s}$ the number of stripes in the simulation box containing $N$ particles. In the same figure we show the prediction of the Luttinger liquid theory (black line), whose comparison with our results hints that the superfluid signal in the stripes is more robust against system size and temperature (encoded in the parameter $\gamma$ ) than what the Luttinger theory predicts for a 1D system. Therefore, we conclude

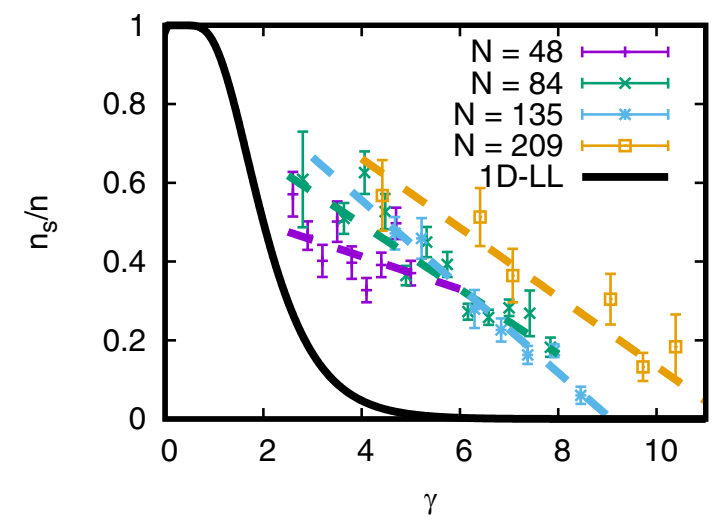

FIG. 4. Superfluid fraction of the stripe phase for different number of particles as a function of the scaling parameter of Luttinger theory $\gamma$ for density $n r_{0}^{2}=128$ and tilting angle $\alpha=0.6$. Solid black line corresponds to the Luttinger liquid prediction of Eq. (6). As it can be seen, there is no collapse of the data to a single line.

that the stripe phase of a two-dimensional dipolar system cannot be considered as an ensemble of one-dimensional Luttinger liquids. This result is in agreement with the analysis of simulation data of the one-body density matrix of the stripe phase at zero temperature [12].

\section{B. One-body density matrix}

To get a deeper insight in the supersolid properties of the stripe phase, we have calculated the one-body density matrix (OBDM),

$$
n_{1}\left(\mathbf{r}_{1}^{\prime}, \mathbf{r}_{1}\right)=\frac{V}{Z} \int d \mathbf{r}_{2} \ldots \mathbf{r}_{N} \rho\left(\mathbf{R}^{\prime}, \mathbf{R}\right),
$$

with $\mathbf{R}=\left\{\mathbf{r}_{1}, \mathbf{r}_{2}, \ldots, \mathbf{r}_{N}\right\}, \mathbf{R}^{\prime}=\left\{\mathbf{r}_{1}^{\prime}, \mathbf{r}_{2}, \ldots, \mathbf{r}_{N}\right\}, \rho\left(\mathbf{R}^{\prime}, \mathbf{R}\right)$ the thermal density matrix, and $Z$ the partition function. As it is well known, in 2D systems there is a condensate fraction only in the $T=0$ limit. This condensate fraction, which means that the system has off-diagonal long-range order, is obtained from the asymptotic constant value of $n_{1}\left(\mathbf{r}_{1}^{\prime}, \mathbf{r}_{1}\right)$ at large distances. For $T \leqslant T_{c}, n_{1}\left(\mathbf{r}_{1}^{\prime}, \mathbf{r}_{1}\right)$ decays with a power law instead, pointing to what is generally termed as quasicondensate. In contrast, for $T>T_{c}$ the decay turns out to be exponential, as it corresponds to a normal phase.

In Fig. 5, we show PIMC results for the OBDM in the stripe phase $\left(n r_{0}^{2}=128, \alpha=0.6\right)$ at different temperatures. Below the BKT transition temperature, the long-range behavior of the OBDM is well captured with a fit of the form $n_{1}(r) \sim r^{-\eta}$. The value of the exponent $\eta$ is given by the BKT theory,

$$
\eta=\left(m k_{B} T\right) /\left(2 \pi \hbar n_{s}\right)
$$

becoming maximal at the critical point, $\eta_{c}=1 / 4$. As we can see in Fig. 5, the algebraic decay of the PIMC results below $T_{c}$ reproduces the $\mathrm{BKT}$ prediction. When the stripes become normal, the OBDM changes dramatically and we clearly see an exponential decay.

\section{Stripe melting}

When temperature is increased beyond $T_{c}$, the stripe phase still persists as the ground state of the system, but as a 


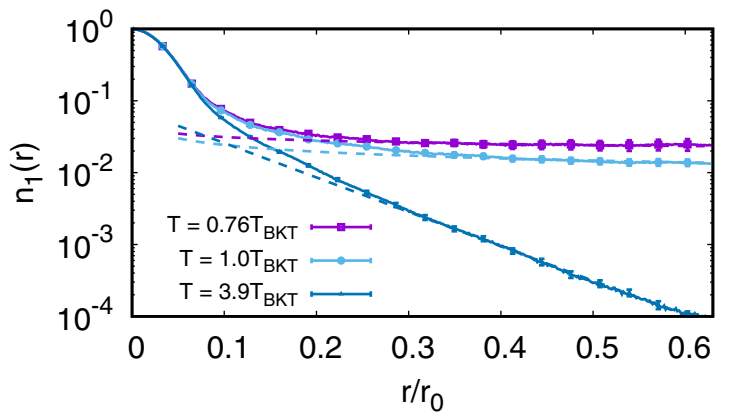

FIG. 5. One-body density matrix of the stripe phase $\left(n r_{0}^{2}=128\right.$ and $\alpha=0.6$ ) at different temperatures, above and below the transition temperature $T_{c}$. The $y$ axis is in log scale and the straight lines correspond to the asymptotic behavior when $r \rightarrow \infty$.

normal phase (nonsuperfluid). Under these conditions, the static structure factor still shows a clear Bragg peak in the transverse direction $(Y)$ pointing to the stability of the stripes [41]. Thus this is an interesting quantity if one wants to estimate the critical temperature at which the stripe phase melts towards the gas one. To study this, we evaluate the static structure factor for wave vectors perpendicular $(Y)$ to the stripe direction $(X)$,

$$
S_{y}(k)=\frac{1}{N Z}\left\langle\hat{\rho}_{-\mathbf{k}_{y}} \hat{\rho}_{\mathbf{k}_{y}}\right\rangle,
$$

with $\hat{\rho}_{\mathbf{k}_{y}}=\sum_{i=1}^{N} e^{i \mathbf{k}_{y} \cdot \mathbf{r}_{i}}$ the density-fluctuation operator. In Fig. 6, we show results of $S_{y}(k)$, for a characteristic point of the phase diagram where the system is in the stripe phase, as a function of the temperature. The Bragg peak that appears at a characteristic $\mathbf{k}_{y}$ signals the periodic pattern of the stripes in their transverse direction. This large peak, which increases with the number of particles $N[38,41]$, is the best signature of the stripe order. When the temperature increases, the strength of the peak decreases due to the increase of the thermal motion. At the largest temperature reported in Fig. 6, the Bragg peak has disappeared pointing to its melting to a gas. Notice that no equivalent peak appears at any $T$ in the $X$ direction.

However, the localization decreases progressively with $T$ until we observe their melting at a temperature $T \simeq 10 T_{\mathrm{BKT}}$.

The evolution of the stripe structure can also be qualitatively analyzed by looking at the spatial distribution of

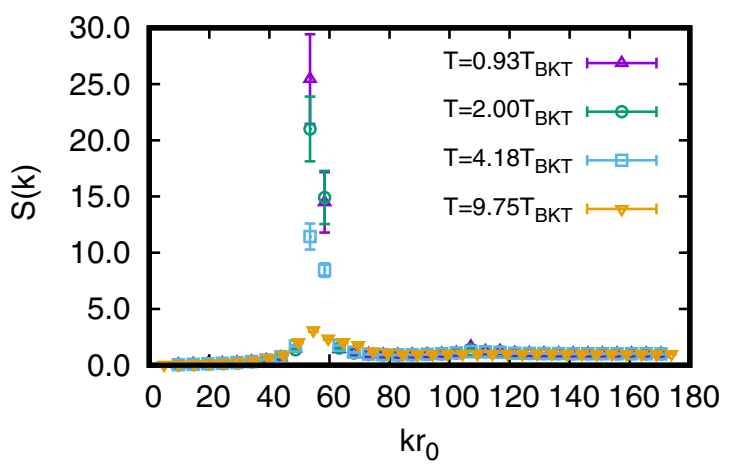

FIG. 6. Evolution with the temperature of the static structure factor $S_{y}(k)$ in the stripe phase at $n r_{0}^{2}=128$ and $\alpha=0.6$.

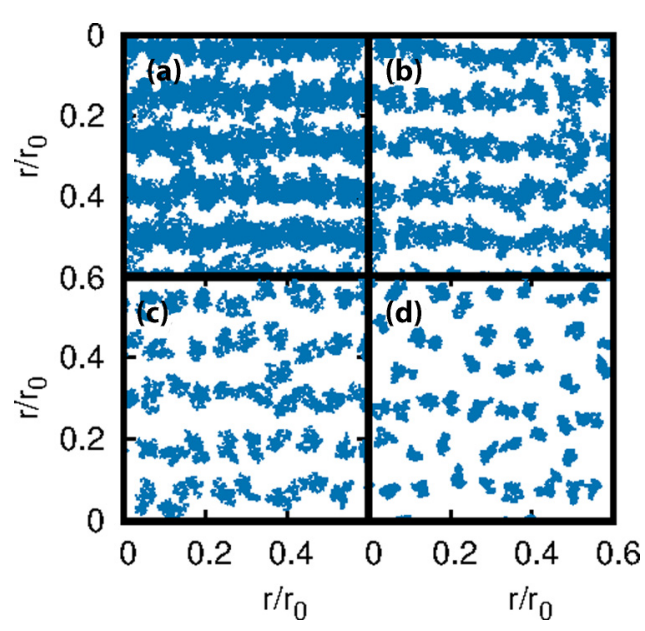

FIG. 7. Snapshots of the PIMC simulations of the stripe phase for increasing temperatures at $n r_{0}^{2}=128$ and $\alpha=0.6$. The temperature $T$ increases from (a) to (d) panels. The values of $T$ are the same as in Fig. 6.

particles in the PIMC simulation. In Fig. 7, we show snapshots to show this evolution with increasing $T$. In the PIMC framework, each particle is represented by a polymer with an averaged size proportional to its quantum delocalization. At temperatures below $T_{c}$, one can see from the snapshots that there are paths connecting the different linear structures (stripes); when these crossing paths are of the length of the simulation box there is a nonzero winding number in that direction and the superfluid fraction is finite. In the second frame of Fig. 7, these transverse paths have nearly disappeared and also in the $X$ direction the interconnections are not very abundant. In the third frame, we still observe the characteristic order of stripes but dislocations between the different lines start to be apparent. This effect has been deeply studied in Refs. $[42,43]$ and now our microscopic simulations confirm these predictions. Finally, the last frame corresponds to a temperature where the stripe structure is no longer present because it has melted to a (normal) gas.

\section{CONCLUSIONS}

In conclusion, we have carried out a complete study of the BKT transition in anisotropic $2 \mathrm{D}$ systems of quantum dipoles. Using the BKT theory we have estimated the superfluid-tonormal phase transition critical temperature at different densities and tilting angles. At fixed density, and increasing the tilting angle, we observe the transition from a gas to a stripe phase with a decrease on the critical temperature in the stripe case. In spite of this reduction, which makes the supersolid phase of stripes less stable against thermal fluctuations than the gas, the superfluid signal is clear below $T_{c}$. The long-range behavior of the OBDM is also consistent with the BKT prediction. Interestingly, our PIMC results on the superfluid fraction show that its value in the transverse direction is still finite but small $(<5 \%)$ close to $T_{c}$ and that at lower temperatures, and mainly close to the transition line to the gas, its value is much larger, almost $100 \%$. This result is qualitatively similar to recent experiments in which a dipolar droplet system, arranged in a quasi-one-dimensional array, has shown superfluid 
signatures across the drops [5-7]. Regarding two-dimensional dipolar systems, similar predictions about the existence of a superfluid stripe phase have been recently reported for the equivalent system in the lattice [13]. Therefore, the quantum dipolar phases seem now the best suited candidates for the realization of the pursued supersolid state of matter. Finally, it is also worth mentioning that a superfluid stripe phase has been studied in the Hubbard model with an isotropic longrange interaction [44]. In this case, the rotational symmetry is broken spontaneously by the interplay between the long-range character of the interparticle interaction considered with the lattice that forces the atoms to occupy certain lattice positions in order to minimize the energy.

\section{ACKNOWLEDGMENTS}

This work has been supported by the Ministerio de Economia, Industria y Competitividad (MINECO, Spain) under Grant No. FIS2017-84114-C2-1-P.
[1] A. F. Andreev and I. M. Lifshitz, Quantum theory of defects in crystals, Sov. Phys. JETP 29, 1107 (1969).

[2] D. Y. Kim and M. H. W. Chan, Absence of Supersolidity in Solid Helium in Porous Vycor Glass, Phys. Rev. Lett. 109, 155301 (2012).

[3] Y. Li, G. I. Martone, L. P. Pitaevskii, and S. Stringari, Superstripes and the Excitation Spectrum of a Spin-OrbitCoupled Bose-Einstein Condensate, Phys. Rev. Lett. 110, 235302 (2013).

[4] J. Léonard, A. Morales, P. Zupancic, T. Esslinger, and T. Donner, Supersolid formation in a quantum gas breaking a continuous translational symmetry, Nature 543, 87 (2017).

[5] L. Tanzi, E. Lucioni, F. Famà, J. Catani, A. Fioretti, C. Gabbanini, R. N. Bisset, L. Santos, and G. Modugno, Observation of a Dipolar Quantum Gas with Metastable Supersolid Properties, Phys. Rev. Lett. 122, 130405 (2019).

[6] L. Chomaz, D. Petter, P. Ilzhöfer, G. Natale, A. Trautmann, C. Politi, G. Durastante, R. M. W. van Bijnen, A. Patscheider, M. Sohmen, M. J. Mark, and F. Ferlaino, Long-Lived and Transient Supersolid Behaviors in Dipolar Quantum Gases, Phys. Rev. X 9, 021012 (2019).

[7] F. Böttcher, J.-N. Schmidt, M. Wenzel, J. Hertkorn, M. Guo, T. Langen, and T. Pfau, Transient Supersolid Properties in an Array of Dipolar Quantum Droplets, Phys. Rev. X 9, 011051 (2019).

[8] S. M. Roccuzzo and F. Ancilotto, Supersolid behavior of a dipolar Bose-Einstein condensate confined in a tube, Phys. Rev. A 99, 041601 (2019).

[9] L. Tanzi, S. M. Roccuzzo, E. Lucioni, F. Famà, A. Fioretti, C. Gabbanini, G. Modugno, A. Recati, and S. Stringari, Supersolid symmetry breaking from compressional oscillations in a dipolar quantum gas, Nature (London) 574, 382 (2019).

[10] M. Guo, F. Böttcher, J. Hertkorn, J.-N. Schmidt, M. Wenzel, H. P. Büchler, T. Langen, and T. Pfau, The low-energy Goldstone mode in a trapped dipolar supersolid, Nature (London) 574, 386 (2019).

[11] G. Natale, R. M. W. van Bijnen, A. Patscheider, D. Petter, M. J. Mark, L. Chomaz, and F. Ferlaino, Excitation Spectrum of a Trapped Dipolar Supersolid and its Experimental Evidence, Phys. Rev. Lett. 123, 050402 (2019).

[12] R. Bombin, J. Boronat, and F. Mazzanti, Dipolar Bose Supersolid Stripes, Phys. Rev. Lett. 119, 250402 (2017).

[13] S. Bandyopadhyay, R. Bai, S. Pal, K. Suthar, R. Nath, and D. Angom, Quantum phases of tilted dipolar bosons in twodimensional optical lattice, Phys. Rev. A 100, 053623 (2019).
[14] V. L. Berezinskii, Destruction of long-range order in onedimensional and two-dimensional systems having a continuous symmetry group I. Classical systems, Sov. Phys. JETP 32, 493 (1971).

[15] J. M. Kosterlitz and D. J. Thouless, Ordering, metastability and phase transitions in two-dimensional systems, J. Phys. C: Solid State Phys. 6, 1181 (1973).

[16] G. Agnolet, D. F. McQueeney, and J. D. Reppy, KosterlitzThouless transition in helium films, Phys. Rev. B 39, 8934 (1989).

[17] D. M. Ceperley and E. L. Pollock, Path-integral simulation of the superfluid transition in two-dimensional He4, Phys. Rev. B 39, 2084 (1989).

[18] M. C. Gordillo and D. M. Ceperley, Path-integral calculation of the two-dimensional 4He phase diagram, Phys. Rev. B 58, 6447 (1998).

[19] P. Minnhagen, The two-dimensional Coulomb gas, vortex unbinding, and superfluid-superconducting films, Rev. Mod. Phys. 59, 1001 (1987).

[20] R. Desbuquois, L. Chomaz, T. Yefsah, J. Léonard, J. Beugnon, C. Weitenberg, and J. Dalibard, Superfluid behavior of a twodimensional Bose gas, Nat. Phys. 8, 645 (2012).

[21] M. Ota, F. Larcher, F. Dalfovo, L. Pitaevskii, N. P. Proukakis, and S. Stringari, Collisionless Sound in a Uniform TwoDimensional Bose Gas, Phys. Rev. Lett. 121, 145302 (2018).

[22] P. A. Murthy, I. Boettcher, L. Bayha, M. Holzmann, D. Kedar, M. Neidig, M. G. Ries, A. N. Wenz, G. Zürn, and S. Jochim, Observation of the Berezinskii-Kosterlitz-Thouless Phase Transition in an Ultracold Fermi Gas, Phys. Rev. Lett. 115, 010401 (2015).

[23] G. Carleo, G. Boéris, M. Holzmann, and L. Sanchez-Palencia, Universal Superfluid Transition and Transport Properties of Two-Dimensional Dirty Bosons, Phys. Rev. Lett. 111, 050406 (2013).

[24] I. Maccari, L. Benfatto, C. Castellani, I. Maccari, L. Benfatto, and C. Castellani, The BKT Universality Class in the Presence of Correlated Disorder, Condens. Matter 3, 8 (2018).

[25] A. Filinov, N. V. Prokof'Ev, and M. Bonitz, BerezinskiiKosterlitz-Thouless Transition in Two-Dimensional Dipole Systems, Phys. Rev. Lett. 105, 070401 (2010).

[26] G. E. Astrakharchik, J. Boronat, I. L. Kurbakov, and Yu. E. Lozovik, Quantum Phase Transition in a Two-Dimensional System of Dipoles, Phys. Rev. Lett. 98, 060405 (2007).

[27] D. M. Ceperley, Path integrals in the theory of condensed helium, Rev. Mod. Phys. 67, 279 (1995). 
[28] M. Takahashi and M. Imada, Monte Carlo Calculation of Quantum Systems, J. Phys. Soc. Jpn. 53, 963 (1984).

[29] M. Takahashi and M. Imada, Monte Carlo Calculation of Quantum Systems. II. Higher Order Correction, J. Phys. Soc. Jpn. 53, 3765 (1984).

[30] S. A. Chin, Quantum statistical calculations and symplectic corrector algorithms, Phys. Rev. E 69, 046118 (2004).

[31] S. A. Chin and C. R. Chen, Gradient symplectic algorithms for solving the Schrödinger equation with time-dependent potentials, J. Chem. Phys. 117, 1409 (2002).

[32] K. Sakkos, J. Casulleras, and J. Boronat, High order Chin actions in path integral Monte Carlo, J. Chem. Phys. 130, 204109 (2009)

[33] M. Boninsegni, N. V. Prokof'ev, and B. V. Svistunov, Worm algorithm and diagrammatic Monte Carlo: A new approach to continuous-space path integral Monte Carlo simulations, Phys. Rev. E 74, 036701 (2006).

[34] D. R. Nelson and J. M. Kosterlitz, Universal Jump in the Superfluid Density of Two-Dimensional Superfluids, Phys. Rev. Lett. 39, 1201 (1977).

[35] J. M. Kosterlitz, The critical properties of the twodimensional xy model, J. Phys. C: Solid State Phys. 7, 1046 (1974).

[36] E. L. Pollock and D. M. Ceperley, Path-integral computation of superfluid densities, Phys. Rev. B 36, 8343 (1987).
[37] A. Macia, J. Boronat, and F. Mazzanti, Phase diagram of dipolar bosons in two dimensions with tilted polarization, Phys. Rev. A 90, 061601(R) (2014).

[38] F. Mazzanti, R. E. Zillich, G. E. Astrakharchik, and J. Boronat, Dynamics of a Two-Dimensional System of Quantum Dipoles, Phys. Rev. Lett. 102, 110405 (2009).

[39] A. MacIa, F. Mazzanti, J. Boronat, and R. E. Zillich, Microscopic description of anisotropic low-density dipolar Bose gases in two dimensions, Phys. Rev. A 84, 033625 (2011).

[40] L. Vranješ Markić, H. Vrcan, Z. Zuhrianda, and H. R. Glyde, Superfluidity, Bose-Einstein condensation, and structure in onedimensional Luttinger liquids, Phys. Rev. B 97, 014513 (2018).

[41] A. Macia, D. Hufnagl, F. Mazzanti, J. Boronat, and R. E. Zillich, Excitations and Stripe Phase Formation in a Two-Dimensional Dipolar Bose Gas with Tilted Polarization, Phys. Rev. Lett. 109, 235307 (2012).

[42] Z. Wu, J. K. Block, and G. M. Bruun, Liquid crystal phases of two-dimensional dipolar gases and Berezinskii-KosterlitzThouless melting, Sci. Rep. 6, 19038 (2016).

[43] A. Mendoza-Coto, D. G. Barci, and D. A. Stariolo, Quantum and thermal melting of stripe forming systems with competing long-range interactions, Phys. Rev. B 95, 144209 (2017).

[44] G. Masella, A. Angelone, F. Mezzacapo, G. Pupillo, and N. V. Prokof'ev, Supersolid Stripe Crystal from Finite-Range Interactions on a Lattice, Phys. Rev. Lett. 123, 045301 (2019). 